\title{
Supplier Selection in Virtual Enterprise Model of Manufacturing Supply Network
}

\author{
Toshiya Kaihara and Jayeola F. Opadiji \\ Kobe University, Graduate School of Engineering, \\ 657-8501, Japan \\ \{kaihara, femi\}@cs.kobe-u.ac.jp
}

\begin{abstract}
The market-based approach to manufacturing supply network planning focuses on the competitive attitudes of various enterprises in the network to generate plans that seek to maximize the throughput of the network. It is this competitive behaviour of the member units that we explore in proposing a solution model for a supplier selection problem in convergent manufacturing supply networks. We present a formulation of autonomous units of the network as trading agents in a virtual enterprise network interacting to deliver value to market consumers and discuss the effect of internal and external trading parameters on the selection of suppliers by enterprise units.
\end{abstract}

Keywords: Virtual enterprise, supply network, supplier selection, multi-agent.

\section{Introduction}

Synergies forged by manufacturing enterprises in order to remain competitive in their market domain often result in the formation of complex supply networks. There are two major ways of analyzing such networks; based on either focusing on the functional units that make up the network or focusing on the interaction between these functional units. The first approach is referred to as function-based analysis while the second is called market based analysis (Vob, 2006). In the market-based analysis of supply network which we employ in this study, attention is paid to the competitive interaction among autonomous enterprises that make up the network. We consider a convergent manufacturing supply network in which every enterprise unit that makes up the network use a combination of input resources to produce their output. Each enterprise will therefore have to source its input from a number of possible suppliers in different markets. Resource contention and different purchasing overheads for all the autonomous units make the task of finding an optimal solution for the multiple supplier selection problems arduous. Introducing some other constraints such as the fill-or-kill constraint in combinatorial auctions make the job all the more difficult (Xia, 2005). Our approach uses a competitive market algorithm to obtain a satisfying solution to resource distribution problem in a convergent supply network. A satisfying solution presupposes a solution that satisfies all the constraints in the optimization 
model and suffices in relation to the objective function (Schwartz, 2002) (Fingar, 2004). The objective of this work is to provide a tactical planning framework for supplier selection in a supply network which will aid in balancing between competitive behaviour among autonomous enterprises and the ultimate goal of value delivery maximization in the network. Competitive interaction in the supply network is provided for in this model by means of $(k+1)$ st auction algorithm which is a variant of the Vickery auction(Vickery, 1961) (Walsh, 2003). In order to enhance value delivery, the network is modeled as a Virtual Enterprise Network (VEN). The concept of a VEN assumes the existence of a supply network consisting of autonomous business units which interact in such a way as to form one giant enterprise with the goal of maximizing value delivery to consumers; their interaction is enabled by means of Information Technology infrastructure (Camarinha-Matos, 1999). We assume a set of enterprise agents that are individually rational and an auction mechanism that is incentive compatible; therefore, going by the impossibility theorem (Myerson, 1983), we can only obtain solutions that trade-off between budget balance and economic efficiency. Our goal at this stage of our work is to obtain solutions with no budget subsidies.

\section{Supply Network Model}

\subsection{Supply Network}

The supply network shown in figure 1 is a convergent manufacturing supply network with resource complementarity; the demand for a particular input resource by an enterprise unit affects the demand of other input resources by the same enterprise unit. As shown in the figure, the generalized supply network is made up of $(l+1)$ layers with layers 1 to $l$ representing the production section of the supply network and layer $C$ representing the consumption layer. In each of the production layers, there are multiple markets with each market containing enterprises that require inputs from a preceding layer to produce a resource unique to that market. It is possible for the number of markets in the layers to be different. The figure can be represented using a graph description as follows:

$$
\begin{aligned}
& S=\text { set of productionenterprise } \\
& C=\text { set of consumers } \\
& H:(N, A) \\
& N=T \cup G=\text { nodes in graph } \mathrm{H} ; T:\{S, C\}=\text { traders; } G=\text { goods } \\
& A:\left\{a_{i}=\langle g, t\rangle_{i} \text { or }\langle t, g\rangle_{i} \mid t \in T ; g \in G\right\}=\text { set of directed arcs in graph } H \\
& \langle g, s\rangle=\text { resource } g \text { is an input toenterprises } \\
& \langle g, c\rangle=\text { resource } g \text { is consumedby consumerc } \\
& \langle s, g\rangle=\text { resource } g \text { is producedby enterprises }
\end{aligned}
$$




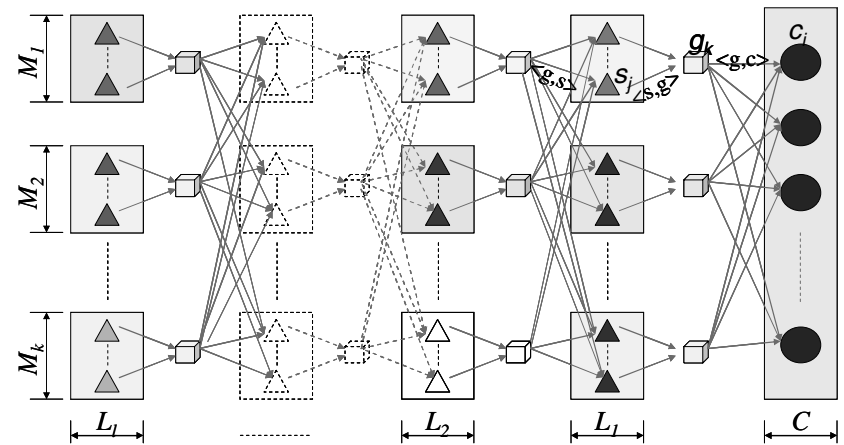

Fig. 1. Convergent supply network with alternative suppliers

\subsection{Supplier Selection Problem}

With the above graph description, the supply network planning problem can be defined with regards to allocation of resources across the network as

$$
u\left(\left(N^{*}, A^{*}\right)\right)=\max _{\left(N^{\prime}, A^{\prime}\right) \in(N, A)}\left(u\left(\left(N^{\prime}, A^{\prime}\right)\right) \mid\left(N^{\prime}, A^{\prime}\right) \text { is feasible }\right)
$$

Given that

$$
u\left(\left(N^{\prime}, A^{\prime}\right)\right)=\sum_{c \in C} u_{c}\left(\left(N^{\prime}, A^{\prime}\right)\right)-\sum_{s \in S} \pi_{s}\left(\left(N^{\prime}, A^{\prime}\right)\right)
$$

s.t $\quad u_{t} \geq 0 ; \quad \forall t \in T$ and $g \in G$

$u_{t}=$ value accrued to an enterprise and $\pi_{s}=$ production cost of supplier $s$

Equation (1) is an objective function defining the goal of value maximization across the supply network. This objective function requires all participating traders to have feasible allocations in the network. The feasibility condition ensures budget balance in the network. Equation (2) the value accrued for a set of possible allocations across the network. Equation (3) represents the assumption of individual rationality among traders in the network which ensures that no trader incurs a deficit in value. With multiple units of every market resource expected to be traded, a scenario in which every trader computes his own optimal bid prices and bid quantities for market resources is assumed.

\section{Market-Based Solution Approach}

Considering the convergent supply network of figure 1, the network is divided into two parts. The first part is layer $C$ consisting of all pure consumers in the network while the second part consists of all the production layers in the network. We assume the second part to be a Virtual Enterprise (VE). The idea of modeling this part of the network as a VE stems from the need to induce cooperation among individually rational competing enterprise units. This idea is plausible because of the increasing 
popularity of electronic auction markets via the internet which create virtual market places. This makes the outcome of trading dependent on the trading mechanism as much as it depends on the bidding tactics of participating traders. In this model, the VEN is divided into a number of virtual markets depending on the number of resources to be traded in the market. The trading mechanism and the bidding tactics of the two different classes of traders in the market are described next.

\subsection{Trading Mechanism}

The fact that enterprise units may have more than one possible supplier for an input type increases the complexity of the already difficult combinatorial problem. We therefore introduce the idea of average supplier overhead per unit input as known variables for each enterprise agent. These values help them make decisions on which supplier and what quantity of a particular market resource to bid for. The Trading mechanism chosen is based on simultaneous ascending price adjustment as used in (Walsh, 2003) where all consumer agents and agents in the market are only allowed to review their bids upward. However, unlike the $S A M P-S B$ protocol used in that work, where bidding is asynchronous, here, the bidding process is synchronous; this is to reduce the communication overhead of the auction mechanism. The algorithm is listed below:

\section{Algorithm}

- $\quad$ Step 1: Initialize all trading agents and virtual markets

- $\quad$ Step 2: Consumer agents send bids at current market price (Adjust bid if not winning)

- $\quad$ Step 3: Enterprise Agents inspect number of winning sales bid

- $\quad$ Step 4: Enterprise Agents check if there is enough inputs to meet winning

sales bid (if not, adjust procurement bid upward and increment price for sales bid)

- $\quad$ Step 5: Auctions compute new market price for all resources and posts bid results privately using the $(k+1)$ st price mechanism

- $\quad$ Step 6: If no bid revision for all agents auction clears else go to Step 2

- $\quad$ Step 7: Terminate Auction

The $(k+1)$ st price mechanism is used at step 5 of the algorithm to compute the current market going price and how much quantity every of a resource every bidder will be allocated at that going price. The traders can then review their bids accordingly if their bidding tactics permit it. A market clearing point is reached when no trader is willing to review their bids at the current going price. The traders are then allocated the quantities of the resource they bid for at their bid prices. It is guaranteed that this algorithm will reach the point of market equilibrium but the equilibrium point may differ from the point of optimality. This difference is a function of the bidding tactics of the trading agents in the market. The advantage of the simultaneous ascending price trading mechanism is that it allows bidding to move in only a single direction 
thereby increasing the speed with which the system reaches equilibrium since bidders can not offer bid prices in both the positive and negative sides of the going price in the market.

\subsection{Bidding Tactics of Traders}

This section is devoted to formulations of bidding tactics of each of the agent types in the network.

\section{Consumers}

A pure consumer is assumed to be a trader whose main interest is to use its endowment of numeraire resource (money) to satisfy a private utility need. The total endowment of consumers represents the total amount of possible investment into the supply network over the planning phase. The bidding tactic of consumer agents in the market is defined as follows:

$$
\begin{gathered}
p_{c}\left(g_{i}\right)=p^{\prime}\left(g_{i}\right)+\alpha_{c} \quad \text { if } p\left(g_{i}\right)<p^{\prime}\left(g_{i}\right) \\
\text { For } \quad g_{i}=\underset{g_{i} \in G}{\arg \max }\left(\min \left(e_{c}^{g_{i}}, p_{c}\left(g_{i}\right)\right)\right) \\
\text { s.t. } \quad\left(e_{c}^{g_{i}}-p_{c}\left(g_{i}\right)\right) \geq 0 ; \quad \forall i \in G ;
\end{gathered}
$$

Where

$$
\begin{aligned}
& p_{c}\left(g_{i}\right)=\text { new bid price of consumer } c \text { for resource } i \\
& p^{\prime}\left(g_{i}\right)=\text { current market price for resource } i \\
& g_{i}=\text { bid quantity for resource } i \\
& \alpha_{c}=\text { price bid adjustment variable of consumer } c \\
& e_{c}^{i}=\text { endowment of consumer } c \text { for resource } i
\end{aligned}
$$

Equation (4) is the price bidding tactic for the consumer agent. A consumer agent adjusts its bid price by a value $\alpha_{c}$ if its last bid price is not enough to make it win all the quantity of that input. It therefore bids above the current market price for that input. Equation (5) represents the quantity of an input a consumer agent will bid for at its current bid value. It bids such that it can get as much units as possible at the current bid price subject to its total valuation for that input. Equation (6) guarantees individual rationality on the part of the consumer agent.

\section{Producers}

Every production enterprise belongs to a virtual market in which it produces. They however require inputs from other markets. This makes them both consumers and producers in the supply network. Their bidding tactic is formulated as:

$$
\begin{gathered}
p_{w}^{i+1}\left(g_{o}\right)=\left\{\begin{array}{l}
\max (A, B) ; \text { if } p_{w}^{i}\left(g_{k}\right)<p^{\prime}\left(g_{k}\right) \\
p^{\prime}\left(g_{o}\right) \\
\text { otherwise }
\end{array}\right. \\
A=\left(p_{w}^{i}\left(g_{o}\right)+\beta_{w}\right)
\end{gathered}
$$




$$
\begin{aligned}
& B=\left(\sum_{k=1}^{m_{l+1}} p_{w}^{i+1}\left(g_{k}\right)+c_{k}^{w}\right) \\
& p_{w}^{i+1}\left(g_{k}\right)=\max \left(p^{\prime}\left(g_{k}\right), p_{w}^{i}\left(g_{k}\right)+\alpha_{k}^{w}\right) \\
& g_{o}=\underset{g_{o} \in G_{L} \subset G}{\arg \max }\left(p^{\prime}\left(g_{o}\right)\right) \\
& g_{k}=\underset{g_{k} \in G_{L-1} \subset G}{\arg \max }\left(\left(p^{\prime}\left(g_{k}\right)+c_{k}^{w}\right)\right) \quad \forall k=1,2 \ldots m_{l-1}
\end{aligned}
$$

s.t.

$$
\begin{aligned}
& \left(\sum_{k=1}^{m_{l-1}} p_{w}\left(g_{k}\right)+c_{k}^{w}\right) \leq p^{\prime}\left(g_{o}\right) \\
& g_{k} \leq \max \left(g_{k}(S P H)\right) \\
& g_{o} \leq \max \left(g_{o}(S P H)\right)
\end{aligned}
$$

where

$S P H=$ strategic planning time span

$p_{w}^{i+1}\left(g_{o}\right)=$ new bid price for output resource of enterprise $w$

$p_{w}^{i+1}\left(g_{k}\right)=$ new bid price for input resource $k$ of enterprise $w$

$p^{\prime}\left(g_{k}\right)=$ current market price of input resource $k$

$\beta_{w}=$ sales bid price adjustment variable of enterprise $w$

$c_{k}^{w}=$ overhead cost of procuring resource $k$ for enterprise $w$

$\alpha_{k}^{w}=$ input bid price adjustment variable of resource $k$ for enterprise $w$

$g_{k}=$ bid quantity of enterprise $w$ for input resource $k$

$g_{o}=$ output resource bid quantity of enterprise $w$

$\max \left(g_{k}(S P H)\right)=$ procurement budget estimate for resource $k$ over strategic planning horizon

$m_{l+1}=$ total number of markets in input layer $(l+1)$

Equation (7) is the price bidding function of an enterprise agent for its product (selling price). It updates this price whenever there is a change in the price of any of its inputs. Equation (8) is a producer's adjusted selling bid price while equation (9) is the adjusted selling price due to variation in the price of an input resource. The price bid for inputs is done in much the same way as in the case of a consumer agent as shown in equation (10). Equation (11) is the output quantity bid policy. Equation (12) is the quantity bid function for inputs and is determined by the number of units the enterprise agent is willing to sell at that point in time. The equation shows how an enterprise agent selects the suppliers of an input by considering the allocation that will 
minimize its average overhead cost, i.e. the most input at the cheapest cost. The constraint of equation (13) is the non-negative profit constraint while equation (14) is the input budget constraint imposed by the strategic plan and equation (15) is the output capacity constraint also imposed by the strategic plan of the enterprise.

\section{Simulation Experiments}

\subsection{Hypothetical Network Simulation}

Experimental simulation was conducted with a hypothetical target supply network (Opadiji and Kaihara, 2008) made up of four layers. The first layer is the consumer layer while each of the three remaining layers is a production layer which consists of more than one market. Figure 2 shows the layout of this hypothetical network. In this network, a resource combination ratio of unity across the network is assumed. This means that every production enterprise requires one unit of all of their inputs to be able to produce their outputs; this is strictly for simplicity and there is no loss of generalization. Also, the bidding process in the virtual enterprise network is assumed to be synchronous and enterprise units bid for resources in bundles rather than in single units.

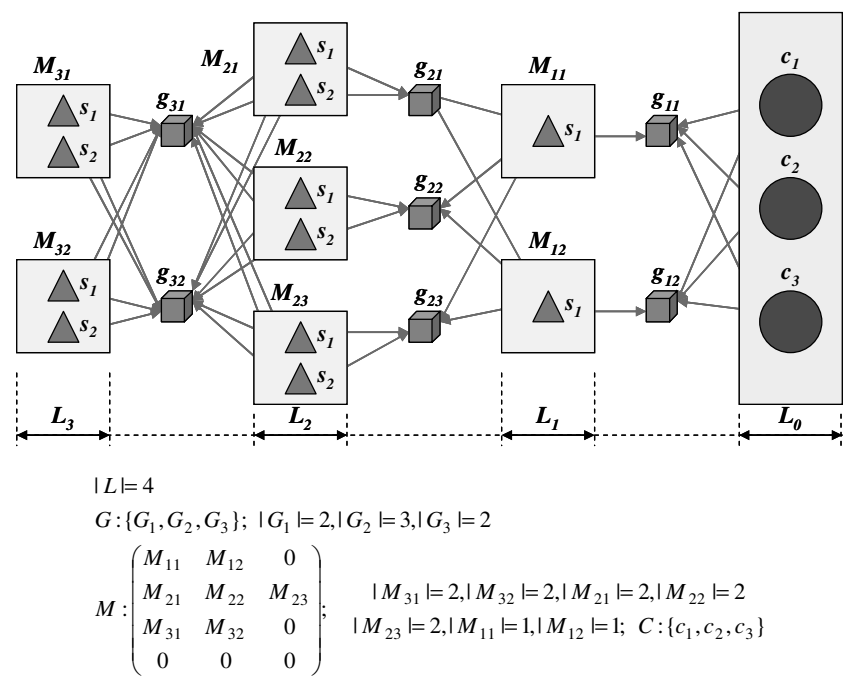

Fig. 2. Target convergent supply network

\subsection{Simulation Results and Discussions}

Initially, a production enterprise bids its maximum output with the hope of securing all the inputs it requires to meet the demand. However, as prices rise in its input markets and it has to bid higher for the inputs, it is possible for it not to be able to secure all its input at the current market price, therefore it drops bid in its output market to the size of the lowest amount of input units it is able to secure. This method prevents 
a producer from winning output bids without being able to secure enough inputs. Hence, the output of producers decrease in response to market states until the market clearing point is reached. Figure 3 shows changes in the output quantity bids of enterprise units in the supply network as trade progresses.

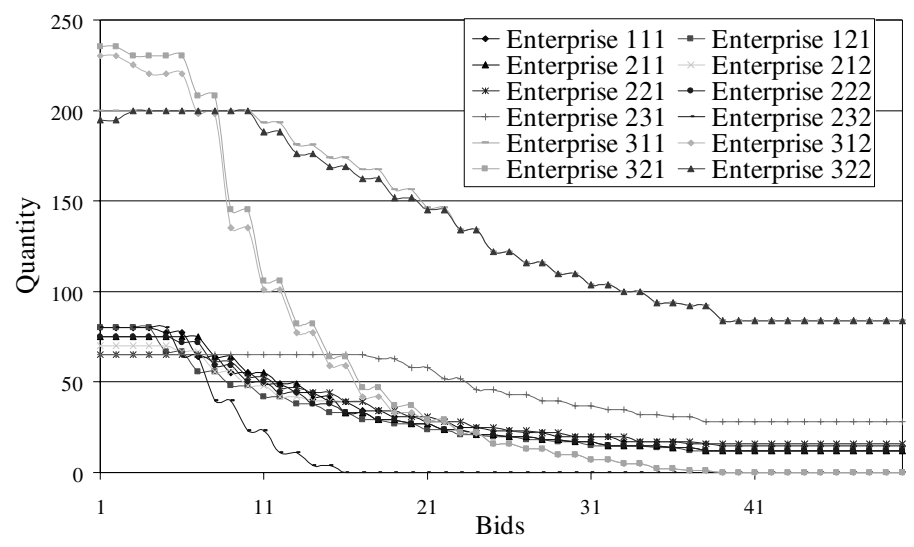

Fig. 3. Supply bids of production enterprises

The bidding tactics of enterprise units are such that they send bids to suppliers via the auction mechanism based on their perceived input and overhead cost as well as their maximum procurement budget for each supplier. If an enterprise needs more inputs than can be supplied by one supplier, it bids for the inputs from other suppliers in order of cost preference. After the market clears, suppliers of all market resources would have been selected and various supply quantities allocated to them by the auctioneers in the virtual enterprise network. Figures 4 and figure 5 show supply and demand allocations for members of the hypothetical supply network generated from simulation.

From figure 4, it is observed that some enterprise units have zero supply allocation. This is because the condition of the virtual markets as dictated by private bidding variables of each trader makes them uncompetitive at the present tactical planning horizon. It then means for example, that only Enterprise 31 of layer $L_{2}$ will supply all the units of market resource 3 of layer $L_{2}$ required by producers in layer $L_{1}$ because Enterprise 32 of the same layer has been eliminated from the allocation while both Enterprise 11 and Enterprise 12 will supply market resource 1 of layer $L_{2}$ because both remain competitive till market clearing. The same holds for all the order layers in the virtual enterprise.

In figure 5, the demand of all consuming traders in the supply network is shown; traders in layer $L_{3}$ are not included because they are assumed to be primary producers transforming resources from their natural state into outputs that can be used by used by enterprises in the next layer. In this figure it can also be observed that producers that are not selected for any supply allocation do not have any input allocated to them. This satisfies the individual rationality assumption in the auction mechanism. Figure 6 depicts link formation in the network. 


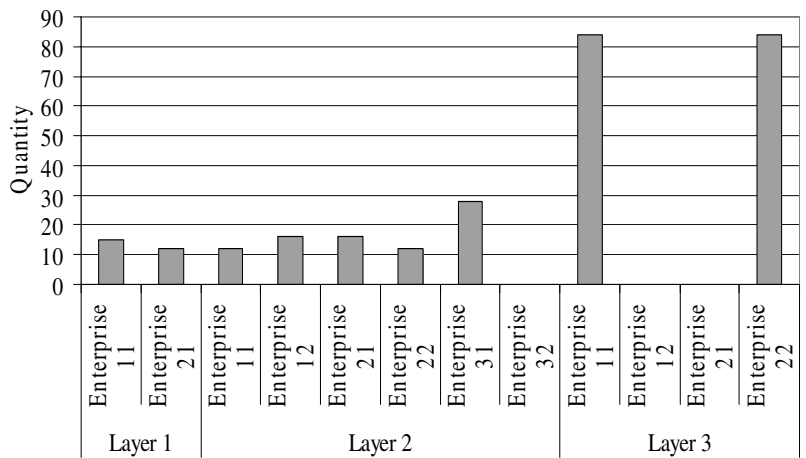

Fig. 4. Supply allocation of producers

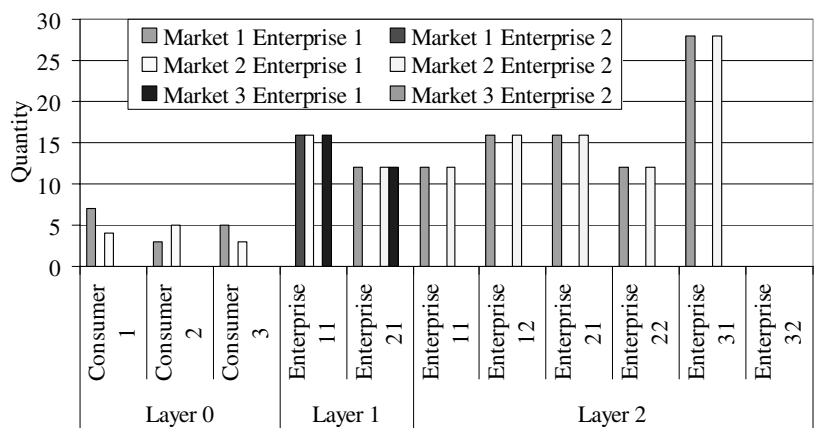

Fig. 5. Demand allocation of all traders

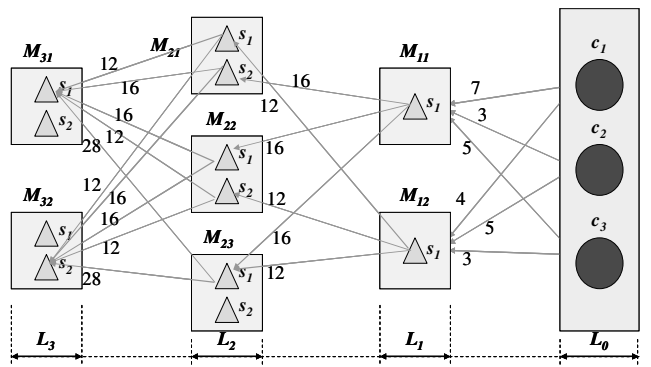

Fig. 6. Supplier selection and resource allocation 


\section{Conclusions}

Supplier selection in a supply network is essentially a tactical level decision which requires compliance with the strategic procurement budgets of all the enterprises in the network. The increasing popularity of internet-based auctions makes it possible for autonomous enterprises to form virtual networks with low information latency that will help increase the flexibility of their value delivery system. Such virtual market places require robust mechanisms that will provide solutions acceptable to all participants where there is contention for resources. We have presented a market-based approach to solving the supplier selection problem in a typical convergent manufacturing supply network. This model obtains a satisficing solution which obtains budget balance at the expense of network efficiency. In this study, some parameters that affect the selection of suppliers by individual enterprise units were considered and the effects they have on the throughput which is a measure of network efficiency. However, a concrete algorithm on how to go about adjustment of some environmental variables using the many auctioneers in the market is still being researched. The complexity of this problem stems from the fact that there are many auctioneers in the virtual enterprise network; therefore a sort of cooperative interaction is required among them. The direction of future research activities will be towards finding correlations between the supplier selection parameters already defined and the effects of mediations by auctioneers on the network throughput and budget balance.

\section{References}

1. Camarinha-Matos, L.M.: The Virtual Enterprise Concept. Infrastructures for Virtual Enterprises, pp. 3-14. Kluwer, Dordrecht (1999)

2. Fingar, P., Belini, J.: The Real Time Enterprise. Meghan-Kiffer Press, Tampa Florida (2004)

3. Opadiji, J.F., Kaihara, T.: Optimal Resource Allocation in Supply Network with Competitive Market Concept. In: Proceedings of the 41st CIRP International Seminar on Manufacturing Systems, CD-ROM (2008)

4. Myerson, R.B., Satterthwaite, M.A.: Efficient Mechanism for Bilateral Trading. Journal of Economic Theory 29, 265-281 (1983)

5. Schwartz, B., et al.: Maximizing versus Satisficing: Happiness is a Matter of Choice. Journal of Personality and Psychology 83(5), 1178-1197 (2002)

6. Vickery, W.: Counterspeculations, Auctions, and Competitive Sealed Tenders. Journal of Finance 16, 8-37 (1961)

7. Vob, S., Woodruff, D.L.: Introduction to Computational Optimization Models for Production Planning in a Supply Chain, 2nd edn. Springer, Berlin (2006)

8. Walsh, W.E., Wellman, M.P.: Decentralized Supply Chain Formation: A Market Protocol and Competitive Equilibrium Analysis. Journal of Artificial Intelligence Research 19, 513 567 (2003)

9. Xia, M., Stallaert, J., Whinston, A.B.: Solving the Combinatorial Auction Problem. European Journal of Operations Research 164(1), 239-251 (2005) 